\title{
Ransom Test Results from a Bi-Lagrange + Remap Explicit Approach
}

\author{
Vazquez-Gonzalez Thibaud $^{1}$, Llor Antoine ${ }^{1}$ \\ ${ }^{1}$ CEA, DAM, DIF \\ 91297 Arpajon Cedex, France \\ thibaud.vazquez-gonzalez@cea.fr; antoine.1lor@cea.fr
}

\section{Extended Abstract}

The physical modeling of two-phase flows has a wide range of industrial and academic applications. However, producing the corresponding numerical schemes is one of the challenging areas of computational fluid dynamics, due to various specific features such as possible non-hyperbolic behaviours, non-conservative equations, imbalanced pressure closures, competing stiff phenomena, and positivity constraints. On many of these aspects, the Ransom water faucet test has emerged as an important benchmark for numerical schemes.

A thorough review on the non-hyperbolic ill-posedness of two-phase flow models can be found in [1], with a focus on the Ransom test. As recently explained [2], the quest for an hyperbolic, physically sensible model is still not solved. The present work aims at providing some evidence that an elementary scheme---here a bi-Lagrange plus remap extension of usual single-fluid Lagrange schemes---can fare very well compared to other presumably more accurate constructions designed to deal specifically with the above mentioned issues. Although this simple approach does not demand the model equations to be hyperbolic---this can be forced for instance by adding the ad hoc Bestion drag term [3],---it strictly preserves the positivity of masses and volume fractions.

The bi-Lagrange plus remap approach we propose consist of a six equation extension of an already published four equation scheme [4] and is described here in 1D. Each time step is made of three sub-steps: a Lagrangian evolution of each fluid on a staggered mesh, a conservative remap on a common mesh, and an instantaneous pressure relaxation closed by the equation of state. It is thus fully conservative, 1 st order in time and 2 nd order in space.

Compared to other published Ransom tests, our results show good compromise between under-and-overshoots and numerical diffusion. In particular, the discretization ensures consistency between the energy equation and the mass and momentum equations so that the isentropic property of the flow is preserved in the Ransom test.

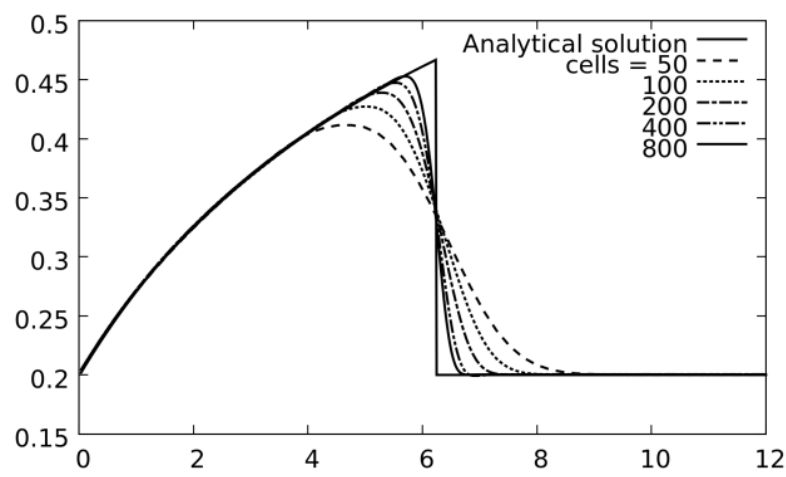

Fig. 1: Profiles of air volume fractions in Ransom test for the 6 equation model.

\section{References}

[1] T. N. Dinh, R. R. Nourgaliev and T. G. Theofanous, "Understanding the ill-posed two-fluid model," NURETH-03, 2003.

[2] D. Lhuillier, C. H. Chang and T. G. Theofanous, "On the quest for a hyperbolic effective-field model of disperse flows," J. Fluid. Mec., vol. 731, pp. 184-194, 2013. 
[3] D. Bestion, "The physical closure laws in the CATHARE code," Nucl. Eng. Des., vol. 124, pp. 229-245, 1990.

[4] P. H. Cournede, Ph.D. dissertation, Ecole Centrale Paris, 2001. 